\section{EBM und medizinische Antilogik}

L. Dubs

Evidence-based Medicine als englischer Begriff kann nur schwer ins Deutsche übersetzt werden; deshalb wird im deutschen Sprachraum häufig einfach von EBM gesprochen. Dennoch möchte ich - mit Hilfe des Dichters Goethe, dessen 250. Geburtstag wir vor kurzem gefeiert haben - eine Deutung des Begriffs «Evidenz» versuchen. Im zweiten Akt, zweiter Aufzug, verkündet Torquato Tasso als Vertreter der Sturmund-Drang-Zeit im gleichnamigen Stück: "Erlaubt ist, was gefällt.» Darauf erwidert die Fürstin Leonore, welche den Beginn der Klassik verkörpert: «Erlaubt ist, was sich ziemt.» Goethe weiterdenkend, möchte ich hier ableiten: «Evidenz (d.h. erlaubt) ist, was dem Patienten nützt.»

Die heutige, naturwissenschaftliche Medizin beruht auf einem kausalen Denkmodell von Ursache und Wirkung. Generationen von Ärztinnen und Ärzten sind in diesem Pathogenesedenken erzogen worden und haben aufgrund solcher Überlegungen Wirkungstheorien erstellen müssen. Das Modell hat seinen Niederschlag in der "International Classification of Diseases" (ICD) gefunden. Diese geht davon aus, dass man etwas von Ätiologie weiss, dass man eine Ahnung davon hat, wie sich bei gewissen Ätiologien Strukturen und Funktionen im Sinne der Krankheitsentstehung (Pathogenese) verändern können und wie sich dies anhand von Symptomen und Befunden manifestieren soll. Am Schluss steht eine Diagnose.

\section{Die ICIDH-Klassifikation}

Seit 1980 existiert zum Glück auch eine andere Klassifikation: die "International Classification of Impairments, Disabilities and Handicaps» (ICIDH) oder zu deutsch: Internationale Klassifikation der Schädigungen, Fähigkeitsstörungen und Beeinträchtigungen. Diese befasst sich mit den Krankheitsfolgeerscheinungen auf drei verschiedenen Ebenen: Auf der Organebene spricht man von Schädigung, auf der Ebene des Individuums von Fähigkeitsstörung und auf der Gesellschaftsebene von Beeinträchtigung. Unabhängig voneinander werden auf diesen drei Ebenen die Schweregrade klassifiziert, werden eigene Namen gegeben und eigene Einteilungen verwendet. Diagnosen entsprechen am ehesten den Schädigungen; man spricht von Sprach- oder Augenschädi-

Korrespondenz:

Dr. med. Luzi Dubs

Merkurstrasse 12

CH-8400 Winterthur gungen, von Schädigungen des Bewegungsapparates oder Schädigungen der Psyche. Bei der Fähigkeitsstörung geht es darum, wie jemand sprechen, hören, sehen kann, ob diese Person sich ankleiden, ob sie essen oder gehen kann. Die Beeinträchtigung drückt aus, ob jemand wegen einer Fähigkeitsstörung in der Gesellschaft benachteiligt ist: Hat er seine Orientierung verloren? Ist er immobil? Ist er ökonomisch abhängig? Ist er sozial desintegriert?

Die Klassifikation auf der Organebene ist relativ einfach, und Befunde können mehr oder weniger wertfrei quantifiziert werden. Auf der Ebene des Individuums und auf der Gesellschaftsebene gewinnen plötzlich Wertsysteme an Bedeutung; es geht vor allem um die "individuelle Wirklichkeit» bzw. um die externe Validität. Zwischen diesen verschiedenen Ebenen besteht denn auch ein gewisses Spannungsfeld. Umgekehrt eröffnet sich die Möglichkeit, auf jeder Ebene zu intervenieren, d.h., zu behandeln. Wenn z.B. eine Schädigung nicht verbessert werden kann, so lohnt sich doch der Versuch, eine Behinderung oder Benachteiligung beeinflussen zu wollen.

Interessant ist dieses Schichtendenken auch für Zycha, einen Kybernetiker (Abb. 1). Er hat das banal formuliert: Physikalische Gesetze können nur dort wirken, wo räumliche Zusammenhänge vorhanden sind, d.h. in horizontaler Ebene zwischen Zelle und Zelle, zwischen Organ und Organ, zwischen Individuum und Individuum, zwischen Gesellschaft und Gesellschaft. Untereinander, in der vertikalen Richtung, gibt es nur Zusammenhänge bzw. funktionale Zusammenhänge. Das ist ein wesentlicher Punkt in unserem Paradigmawechsel, dass wir vom «Ursachen denken" besser weggehen in Richtung "Zusammenhänge denken».

\section{Vom Nutzen in der Medizin}

Die zentrale Definition vom medizinischen Nutzen sehe ich vor allem im Fähigkeitsgewinn oder allenfalls im Fähigkeitserhalt. Es ist entscheidend, was der Patient kann, was er will und was er braucht. Man darf gut und gerne postulieren, dass der Patient der Experte ist in Bezug auf seine Fähigkeiten. Auf der Organebene sind die Ärzte die Experten, aber auf der Individuumsebene ist und bleibt der Patient der Experte.

So ist es denn auch möglich, durch gewisse Massnahmen Fähigkeitsgewinn zu erzielen, auch ohne die Organschädigung direkt beeinflussen zu können und dies bedeutet Evidenz. Ähnliches gilt auch auf dem Niveau der Gesellschaft.

\section{Kurative Logik vs. Antilogik}

Was bedeutet die ICIDH-Klassifikation für die Praxis? In den Abbildungen 2-6 liegen die drei Ebenen übereinander, jede mit einer Schweregradeinteilung von 0 bis 100. In unserer medizinischen kausalen Logik sind wir immer recht gut zufrieden, wenn, wie in 


\section{Abbildung 1}

Schematische Darstellung von den Schichtungen der Natur (Ausschnitt) nach Zycha.

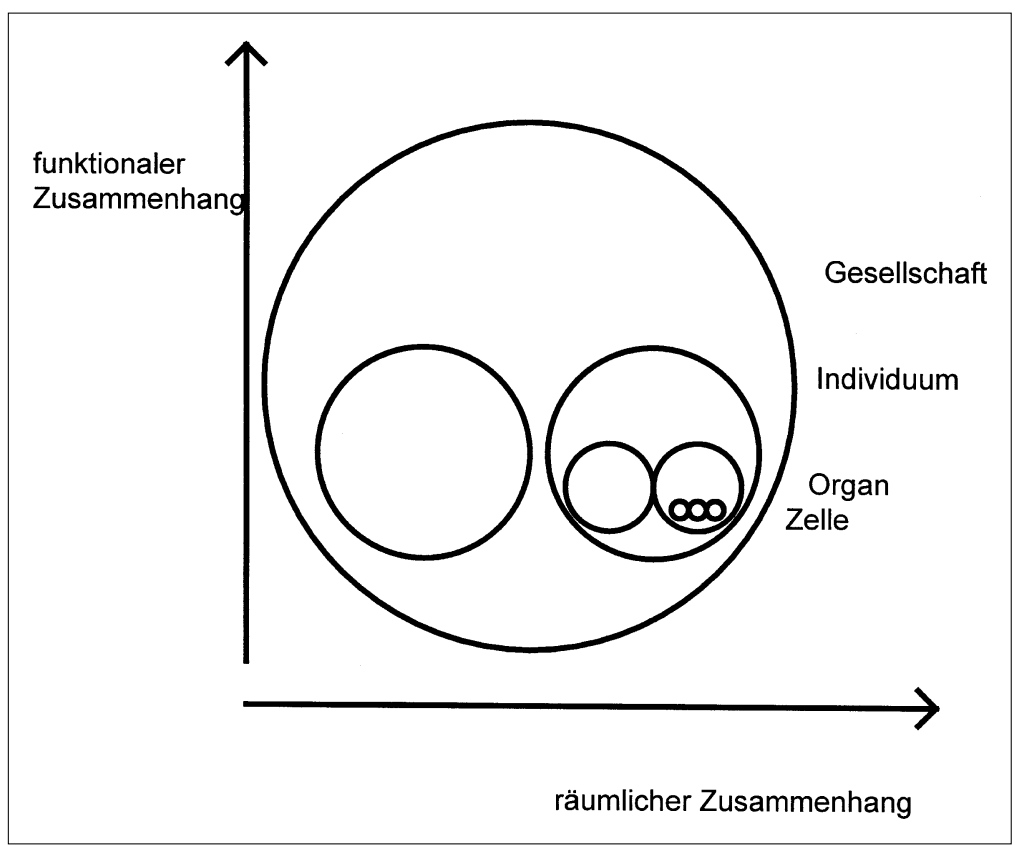

Abbildung 2, der Schweregrad der Organschädigung dem Schweregrad der Fähigkeitsstörung bzw. der Benachteiligung entspricht.

Noch besser fühlen wir uns, wenn wir, wie in $\mathrm{Ab}$ bildung 3, eine Organschädigung verbessern können und gleichzeitig die Fähigkeitsstörung entscheidend abnimmt und die Benachteiligung sich vermindert. Das ist die kurative Logik. Es gibt aber zahlreiche Situationen, in welchen wir an der Schädigung selber nichts mehr verbessern können. Der Schaden bleibt, aber es gibt Möglichkeiten, Fähigkeitsstörungen zu vermindern, und wir können dem Patienten so Nutzen bringen (Abb. 4).

Es kommt nicht darauf an, ob man jetzt von einer Inkongruenz dieser Ebenen ausgeht (Abb. 5) oder, wie in Abbildung 4 sichtbar, von der Kongruenz. Ich möchte damit lediglich andeuten, dass in der Praxis jeweils eher Inkongruenzen vorliegen, welcher Art auch immer. Das ist letztendlich auch die ärztliche Kunst, wie man diese individuelle Wirklichkeit erfasst, wie man diese Punkte setzt.

Wir können durchaus von einer medizinischen Antilogik (Abb. 6) sprechen, wenn wir diese überkreuzte Konstellation haben. Es gibt Menschen mit geringer Organschädigung, aber deutlicher Fähigkeitsstörung und, wenn wir genau hinschauen, vielleicht auch einer erheblichen Benachteiligung. Gelegentlich pflegen wir diesen Menschen zu sagen: «Sie haben ja gar nichts, sie sind gesund.» Der Patient geht nach Hause, aber die Probleme sind nicht gelöst.

Auf der anderen Seite gibt es Patienten mit deutlicher Schädigung, jedoch wenig Fähigkeitsstörungen; diese sind in ihrem sozialen Umfeld sehr gut aufgehoben. Was sagen wir in einem solchen Fall, wenn wir sie überhaupt zu sehen bekommen? «Es ist ja viel schlimmer, als sie denken.» Man ist geneigt, von einer medizinischen Antilogik zu sprechen.

\section{Fallbeispiel (Abb. 7)}

Eine 20jährige Patientin leidet seit dem 8. Lebensjahr an vorderen Knieschmerzen, nebst Asthma und Migräne. Sie kann seit einigen Jahren nicht während längerer Zeit sitzen, nur erschwert Treppen hinuntergehen und keinen Sport betreiben. Sie wurde dreimal arthroskopiert, und jedes Mal sagte man ihr, «sie habe gar nichts, der Knorpel sehe schön aus». Aufgrund dieser Inkongruenz musste man etwas weiter gehen, und nach intensivem Gespräch ergaben sich Hinweise auf eine enorme sogenannte sensorische Schädigung, welche darin bestand, dass die Patientin jahrelang, seit dem 8. Lebensjahr vom alkoholsüchtigen Nachbarn sexuell vergewaltigt wurde, ohne dass jemand davon Kenntnis besass. Die sensorische Schädigung kann durchaus zum beschriebenen Fähigkeitsverlust geführt haben. Da der Knorpel schön aussieht, nützen entlastende Operationen nichts. Die sensorische Schädigung ist in diesem Fall Ausdruck unseres Gesellschaftsbildes, über Jahre hinweg so entwickelt. In Anlehnung an Raspe glaube ich auch, dass die Chronifizierung von Schmerzen Ausdruck der Traumatisierungen in den "life events», in den biographischen Schädigungen ist. Somit erscheint es sinnvoll, die sensorische Schädigung auf der Organebene anzusiedeln. Wer durch Schmerzen behindert ist, macht automatisch weniger, hat somit einen Fähigkeitsverlust, welcher den Schweregrad der Schmerzen widerspiegeln kann.

Ähnlich unangenehm sieht es bei vielen Gastarbeitern aus, die schlechte Rahmenbedingungen haben, keine Schulbildung aufweisen, im Arbeitsmarkt schlecht dastehen, unsere Sprache schlecht verstehen und daher soziale Rehabilitationshindernisse vorfinden. Inkongruenzen in den drei ICIDHEbenen können dadurch erheblich ausgeprägt erscheinen, besonders wenn Ängste als zusätzliche sensorische Schädigung hinzukommen. Gerne lassen wir uns angesichts der Diskrepanzen zwischen Organschädigung und Fähigkeitsstörung dazu verleiten, von Simulanten und Rentenneurotikern zu sprechen. Dabei wissen wir oft zuwenig, was alles an Schädigung in diesen Menschen passiert ist - oder um mit Max Frisch zu sprechen: «Geholt haben sie Arbeitskräfte, gekommen sind Menschen!"

\section{Konsequenzen für die Orthopädie}

Die Orthopädie ist darauf angewiesen, Fähigkeiten als körperliche Leistungsfähigkeit einzuschätzen und als Mass zu nehmen für Nutzen oder Evidenz. Wir können davon ausgehen, die Fähigkeitsleiter des Menschen habe eine gewisse Anzahl Stufen, welche geeignetermassen in Beziehung zur Maslowschen Bedürfnishierarchie gestellt werden können. Zuoberst 


\section{Abbildungen 2-6}

Funktionale Zusammenhänge in den ICIDH-Ebenen.

\section{Abbildung 2}

Medizinische, kausale Logik.

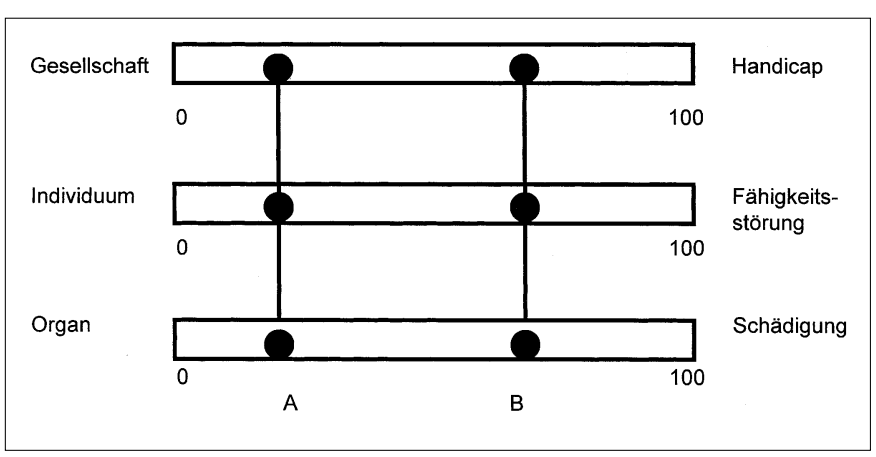

\section{Abbildung 3}

Kurative Logik.

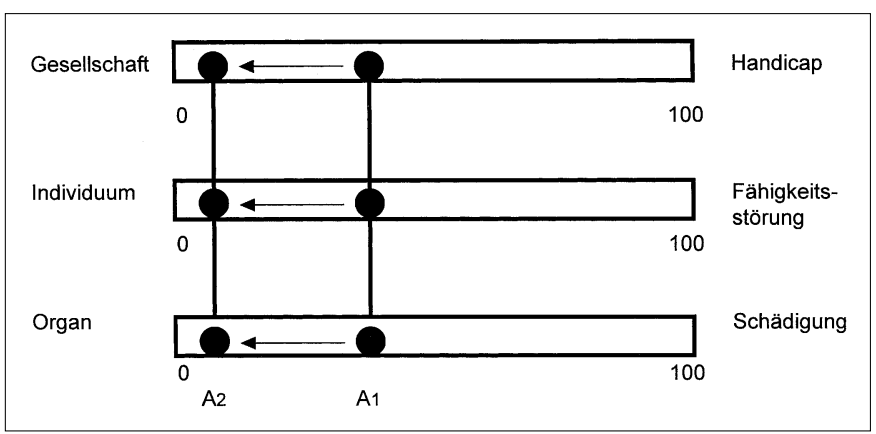

\section{Abbildung 4}

Rehabilitative Logik.

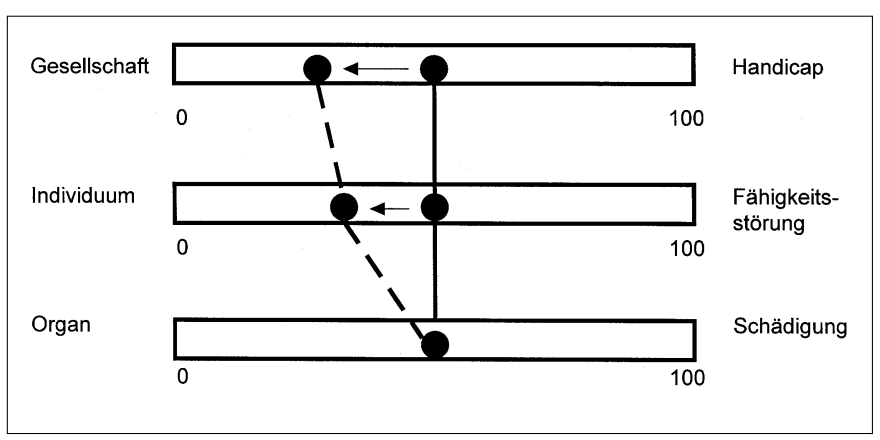

\section{Abbildung 5}

Rehabilitative Logik.

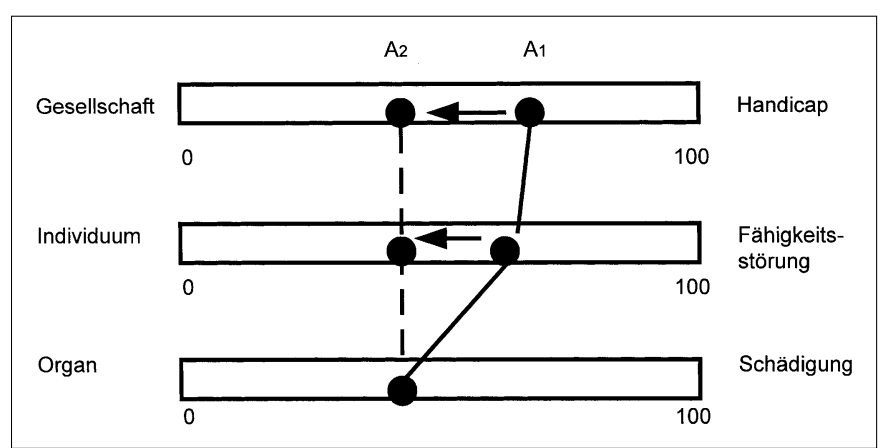

\section{Abbildung 6}

Medizinische Antilogik.

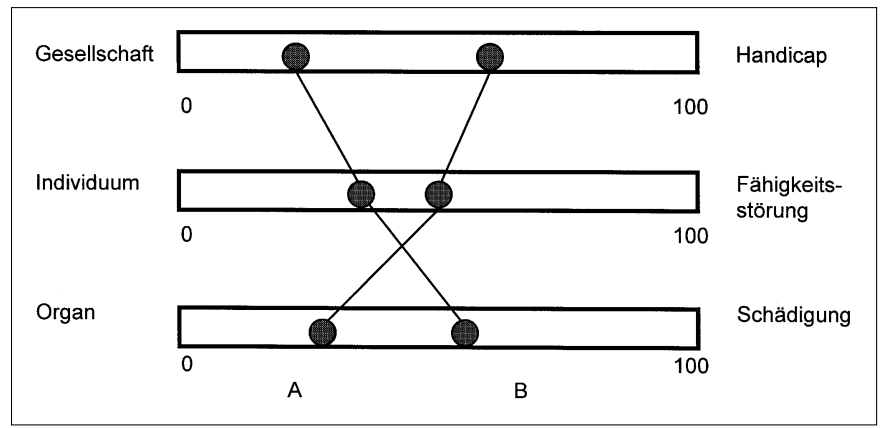

\section{Abbildung 7}

Fallbeispiel: 20jährige Frau mit femoropatellärem Schmerzsyndom (1); der retropatelläre Knorpel ist unauffällig (2), hingegen besteht eine sensorische Schädigung in «life events» (3).

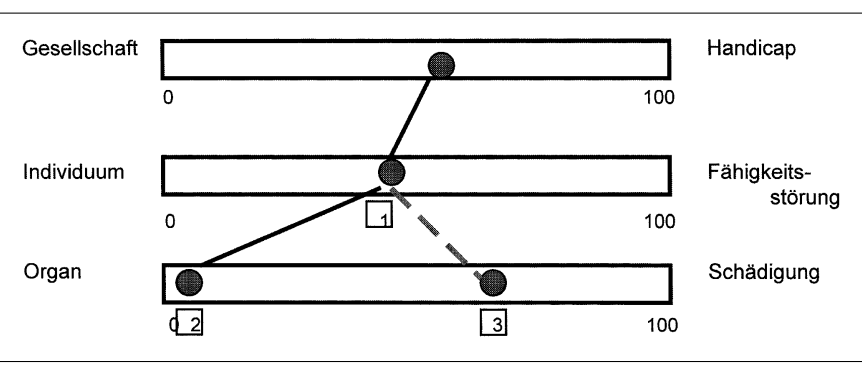


liegt die orthopädische "Selbstverwirklichung», d.h. die heute so bedeutungsvolle Sportfähigkeit. Darunter, nicht immer scharf abzutrennen, die Arbeits- bzw. Berufsfähigkeit. Danach kommen die häuslich-familiären Aspekte der Alltagsfähigkeit, am zentralsten für das Individuum ist jedoch die Selbständigkeit. Diese Fähigkeitsleiter wird in Beziehung zur Zeit gesetzt, so dass sich hypothetisch die "mara»-Kurve ("mean age related ability" oder: mittlerer, altersabhängiger Fähigkeitsverlust) einzeichnen lässt (Abb. 8). In diesem Raster können individuelle Verlaufskurven von Fähigkeitsveränderungen aufgezeichnet werden, um am Einzelfall sowie an Kollektiven den Nutzen der durchgeführten oder unterlassenen Intervention aufzuzeigen. Im Sinne einer Outcome-Studie habe ich an meinem Krankengut von Kreuzbandverletzten einen Fähigkeitsvergleich zwischen operierten und nichtoperierten Patientinnen und Patienten durchführen lassen und erkennen müssen, dass die Kreuzbandoperierten eher schlechter dastehen als die Nichtoperierten bezüglich Fähigkeitsverlauf. Auch wenn diese Hypothese noch weiterer präziserer Studien bedarf, hat diese Erkenntnis von Evidenz bei mir zur Einsicht geführt, dass ich die früher häufig praktizierte Kreuzbandersatzoperation aufzugeben habe, solange ich nicht in der Lage bin, den Nutzen patientengerechter nachzuweisen. Die Wirkungstheorie der mechanistisch geplanten Stabilisierung hat wiederum eine gewisse Antilogik gezeigt, welche erst bewusst geworden ist, wenn man sich die eigentlichen Evidenzfragen stellt, die ICIDH als Denkgrundlage konsequent verwendet und die Möglichkeiten nutzt, welche uns die Methodik der klinischen Epidemiologie zur Überprüfung unseres Wissens offeriert.

\section{Literatur}

Dubs L. Alles getan - Patient noch nicht zufrieden: Einführung in eine Evidence Based Surgery. Swiss Surgery 1999;5:160-6. Maslow AM. Motivation und Persönlichkeit. Hamburg: Rowohlt 1994.

Raspe HH. Mindestanforderungen an das ärztliche Gutachten zur erwerbsbezogenen Leistungsfähigkeit von Kranken mit chronisch-unspezifischen Schmerzen. Versicherungsmedizin 1997;49(4):118-25.

von Uexküll T. Was ist und was will «Integrierte psychosomatische Medizin?» In: von Uexküll T (Hrsg.). Integrierte Psychosomatische Medizin. 3. Auflage. Stuttgart, New York: Schattauer; 1994. S. 17-34.

World Health Organisation. International Classification of Impairments, Disabilities and Handicaps (Übersetzung: Matthesius RG). Berlin, Wiesbaden: Ullstein Mosby; 1995.

Zycha H. Theorie und Erfahrung in der Medizin: Orthodoxe Wissenschaft und ganzheitliche Kybernetik. Forsch Komplementärmed 1998;5 (Suppl. 1):52-9.

\section{Abbildung 8}

Altersabhängige, körperliche Leistungsfähigkeit.

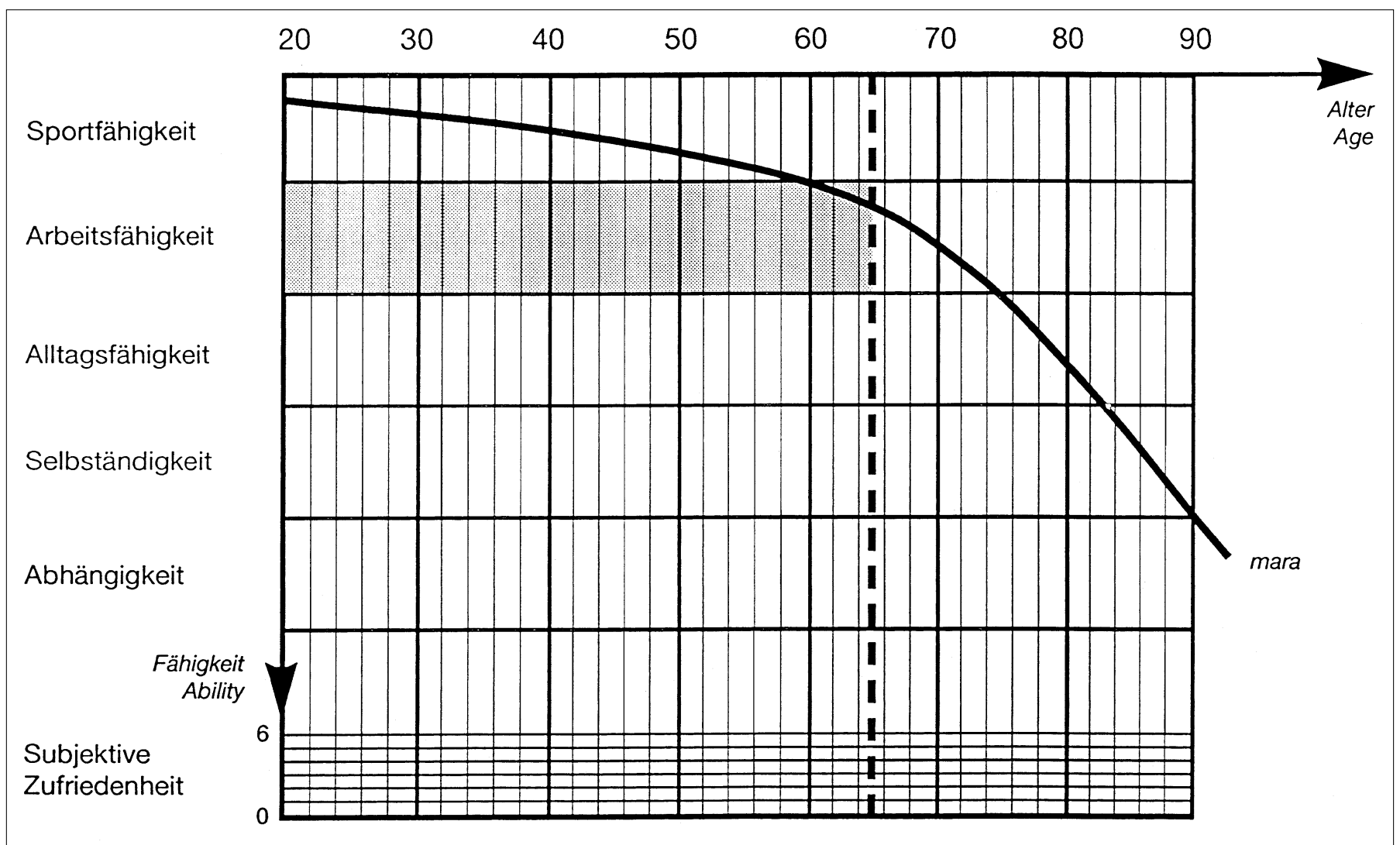

